\title{
REVISIÓN SISTEMÁTICA DE LITERATURA: EVOLUCIÓN DEL ESPACIO ÁULICO EDUCATIVO EN EL CONTEXTO COVID-19
}

\author{
SYSTEMATIC LITERATURE REVIEW: \\ EVOLUTION OF EDUCATIONAL CLASSROOM \\ SPACE IN THE CONTEXT OF COVID-19
}

doi: $10.21555 /$ rpp.v33i33.2414

Uriel Luévano Hernández

Universidad Panamericana, Escuela de Pedagogía,

Campus Aguascalientes, México

0217681@up.edu.mx

https:/ / orcid.org/0000-0002-9604-5857

Leticia Nayeli Ramírez Ramírez

Universidad Panamericana, Escuela de Pedagogía,

Campus Aguascalientes, México

nramirez@up.edu.mx

https:// orcid.org/0000-0002-8113-2368

Recibido: octubre 15, 2021 - Aceptado: noviembre 7, 2021

\section{RESUMEN}

La concepción, el diseño y la construcción del espacio áulico en la educación han experimentado transformaciones a lo largo de la historia; se ha transitado de un espacio tradicional en el que se privilegiaba la funcionalidad, el asoleamiento, la ventilación y la escala, a otro diseño -bajo el contexto COVID-19- en el que se prioriza la flexibilidad, el recálculo de aforos, circulaciones de aire y espacios abiertos. El objetivo de esta investigación es realizar una revisión sistemática de literatura que contemple las investigaciones empíricas sobre la configuración del espacio áulico en la educación, durante el período de 2015 a 2021.

Como criterios de inclusión se tuvieron en cuenta los siguientes: artículos que abordan el tema del espacio áulico de aprendizaje; publicaciones entre 2015-2021, y las realizadas en el contexto de Iberoamérica. Se utilizaron las bases de datos de acceso abierto SCIELO, REDALYC y motores de búsqueda como INVENTIO. En total se recopiló una base de datos de 111 artículos de los cuales se filtraron, sustentados en los criterios de inclusión, 18 artículos 
seleccionados. Los resultados se analizaron con base en la estrategia de análisis de contenido. Se identificaron ocho de las investigaciones de España, tres de México, tres de Colombia, una de Perú, una Ecuador, una de Venezuela y una de Panamá. Se concluye la necesidad de crear espacios áulicos que cumplan con los criterios de aforo limitado y ventilación, para el contexto COVID-19, sin descuidar el empleo de estrategias que promuevan el aprendizaje colaborativo.

Palabras clave: aula, educación, aprendizaje, pandemia, tecnología de la información.

\begin{abstract}
The conception, design, and construction of classroom space in education has undergone transformations throughout history; it has moved from a traditional space in which functionality, sunlight, ventilation, and scale were privileged, to another design under the COVID-19 context in which priority is given to flexibility, recalculation of capacity, air circulation and open spaces. The objective of this research is to conduct a systematic literature review that contemplates empirical research on classroom space configuration in education during the period from 2015 to 2021. The following were considered as inclusion criteria: articles addressing the topic of the classroom space for learning; publications between 20152021 and conducted in the context of Ibero-America. The open access databases SCIELO, REDALYC and search engines such as INVENTIO were used. In total, a database of 111 articles was compiled, from which 18 selected articles were filtered based on the inclusion criteria. The results were analyzed based on the content analysis strategy. Eight of the research were identified from Spain, 3 from Mexico, 3 from Colombia, 1 from Peru, 1 from Ecuador, 1 from Venezuela and 1 from Panama. It is concluded that there is a need to create classroom spaces that meet the criteria of limited capacity and ventilation for the COVID-19 context without neglecting the use of strategies that promote collaborative learning.
\end{abstract}

Keywords: Classroom, Education, Learning, Pandemics, Information Technology.

\title{
INTRODUCCIÓN
}

En el contexto de la pandemia por COVID-19, se modificaron las prácticas de enseñanza-aprendizaje y el espacio áulico en el que tenían lugar. Según cifras proporcionadas por INEGI (2021), en la encuesta para la medición del impacto del COVID-19 en la educación para el ciclo escolar, se inscribieron 32.9 millones de personas entre los 3 y 29 años, y 2.3 millones de personas no están inscritos en el ciclo escolar vigente, por motivos asociados al COVID-19. Dichas cifras reflejan una arista de la desigualdad educativa que se experimenta en México; en promedio, el 85\% de los encuestados está llevando el proceso de enseñanza a distancia, a diferencia de las regiones más pobres, donde solo el $64 \%$ realiza este proceso.

En este tenor, son diversas las fuentes que señalan los factores que incrementan la desigualdad educativa en México durante la pandemia, entre ellos se encuentran: problemáticas económicas, brecha digital, factores pedagógicos, calidad de los aprendizajes, disposición de espacios áulicos, abandono escolar, entre otros.

Señala que los tres factores que contribuyen al impacto de la educación superior en México, derivado del COVID-19, son la desigualdad educativa, abandono escolar y el déficit de aprendizaje. Se invita a realizar estrategias académicas para la impartición de clases a distancia y por medios digitales (Ibero, 2021; Schmelkes, 2020). 
Las políticas públicas internacionales que se han promovido para contrarrestar la desigualdad educativa, apuntalan al regreso a las aulas; dicha política incluye, como lineamientos, la modificación del espacio áulico para la disposición de aprendizajes, a saber: proveer de un espacio seguro con los protocolos de sanidad; crear una rutina y sentido de normalidad en las aulas; adaptar los espacios para ampliar la ventilación; crear espacios flexibles y fluidos que promuevan la colaboración en el aula; adaptar dispositivos de audio y video para clases en modelo híbrido, y utilizar superficies en el mobiliario de fácil limpieza (Steelcase, 2021; UNICEF, 2021).

En este tenor, el regreso a las aulas en el contexto post-COVID, trae consigo diversas reflexiones y adaptaciones en diferentes tópicos de la educación, relativas a los espacios físicos para promover el aprendizaje, las estrategias de enseñanza-aprendizaje, la participación de la familia, y el apoyo emocional. Respecto del espacio físico para el aprendizaje, el uso de Tecnologías de la Información y Comunicación (TIC) -tanto en los centros educativos como en las familias mexicanas- presenta una brecha digital, en la que, hasta 2018, solo $45 \%$ de los mexicanos tenía acceso a un ordenador y 53\% tenía acceso a internet en casa (Lloyd, 2020).

Por tanto, es importante comprender cómo los centros educativos están dando respuesta al uso de los espacios áulicos, los retos a los que se enfrentan y las estrategias que despliegan para la optimización de recursos. El presente artículo de revisión de literatura, tiene como objetivo analizar las investigaciones empíricas sobre configuración del espacio áulico en la educación, durante el contexto COVID-19. A continuación se explican los referentes teóricos que guiaron la investigación.

\section{MARCO TEÓRICO}

\section{Evolución del espacio áulico}

Desde las primeras décadas del siglo XXI, la concepción del aula como espacio de aprendizaje, privilegiaba la funcionalidad, el asoleamiento, la ventilación y la escala; para atender las necesidades pedagógicas e higienistas se dio pauta para que, en la construcción de los nuevos edificios académicos, se aplicaran los principios funcionalista y racionalista.

El aula es concebida como una planta rectangular en proporciones que van de 2:1 y donde la disposición del acomodo de los alumnos surge de forma rígida, paralela al docente, con poca flexibilidad, prevaleciendo la jerarquía del educador sobre el alumno, cuidando la armonía y repetición de los elementos constructivos, y buscando la primicia de dotar espacios de aprendizaje con iluminación y ventilación naturales.

Para Méndez Escobar (2021) es importante el rediseño de las aulas tradicionales hacia nuevos diseños arquitectónicos flexibles y que faciliten el proceso de enseñanza-aprendizaje, mediados por las TIC existentes y los nuevos avances tecnológicos, cuidando también la iluminación, la acústica y la ventilación, además del rediseño del mobiliario y equipo con mayor movilidad y la consideración de espacios áulicos abiertos.

Se sugiere que los docentes participen en el proceso de diseño de la organización del espacio en el aula inteligente, cuando esta sea acondicionada en función de los contenidos curriculares determinados, buscando en todo momento la flexibilidad del espacio y la accesibilidad a los dispositivos y las herramientas tecnológicas, y la versatilidad de la disposición del mobiliario. Se busca que esta flexibilidad favorezca la interacción en un contexto sociocultural, favoreciendo la utilización de las nuevas tendencias pedagógicas emergentes (Mohamed Al-Lal, 2021). 


\section{Modificaciones áulicas para la enseñanza bajo el modelo híbrido}

Dentro de la virtualidad del aula, destaca que los aspectos físicos ambientales, la motivación, la construcción del conocimiento y su consolidación se dan dentro del aula, por lo que aplicarlo a la virtualidad trae consigo ciertos problemas; es por esto que el docente se apoya utilizando tecnologías, pero no es suficiente para trabajar en aulas virtuales (González, Herrera, Clerque y Álvarez, 2020).

Menciona Fernández (2020) que, frente al COVID-19, es de suma importancia el rediseño de los espacios educativos, considerando el recálculo de los aforos, las circulaciones y la accesibilidad universal. Además, se recomienda el uso de los espacios abiertos como gimnasios, salones o bibliotecas para tomar clase, tomando en cuenta: la ventilación para la recirculación del aire y control térmico, la modificación de la iluminación y los protocolos de limpieza y sanitización.

Según Cabrero-Almenara y Martínez-Gimeno (2019), con la implementación de las TIC, la capacitación en el uso tecnológico en el aula y en la docencia, el enfoque pedagógico podría contribuir a mejorar la calidad y el rendimiento académico. Esta pandemia obliga a pensar en aprendizajes híbridos, donde el aula se extiende hasta el hogar del docente y del estudiante, adecuándose -de forma improvisada- los elementos técnicos y tecnológicos para recibir y enviar información vía la red, así como para establecer una interacción con el grupo de forma virtual; es aquí que nace la inquietud de analizar los componentes estudiados sobre el espacio de aprendizaje.

Derivado de la pandemia, se ha implementado un modelo educativo emergente -apoyado en el uso de las aulas híbridas- que, en esencia, es la unión o el aprovechamiento de la tecnología con la presencialidad, atendiendo a la mayor cantidad posible de estudiantes y que permite la flexibilización de la comunidad educativa dentro de la nueva normalidad (Prince, 2021).

Bajo el contexto actual -en el que se continúa con el programa de vacunación del COVID-19 a adultos, jóvenes, niños y niñas-, empieza ya el regreso paulatino al aula física, por lo cual, es importante analizar las estrategias planteadas a nivel nacional, de continuar con el aula virtual y reforzar el aprendizaje híbrido, apoyado por la mediación de las TIC y la reconversión del espacio áulico, teniendo en cuenta los nuevos lineamientos de ventilación, iluminación y flexibilidad.

\section{MÉTODO}

Atendiendo al objetivo de indagar en las investigaciones empíricas sobre la configuración del espacio áulico en la educación durante el contexto COVID-19, se desarrolló la metodología de revisión sistemática de literatura Kitchenham et al. (2010), como un estudio integrativo, sistemático, y de análisis cuantitativo y cualitativo, sobre las preguntas de investigación. Las revisiones sistemáticas proveen una síntesis racional de la investigación básica que supera las limitaciones de las revisiones narrativas, al aplicar estándares rigurosos a la investigación (Beltrán, 2005).

Por su parte, la metodología de revisión sistemática de literatura se clasifica, según el alance y objetivo que se pretenda cubrir, a saber:

a) Revisiones descriptivas: incluyen una revisión que examina el estado de literatura respecto de una pregunta de investigación, un área temática o un concepto.

b) Revisiones de comprobación: buscan responder una pregunta o probar una hipótesis específica, a través de análisis estadísticos o la integración de análisis cuantitativo y cualitativo (Xiao y Watson, 2019). 
La presente investigación se adscribe a la categoría de revisión sistemática de literatura descriptiva, específicamente a través de una revisión de alcance, la cual tiene como objetivo extraer datos relevantes de cada unidad revisada (tanto en la metodología, los hallazgos y las variables) para proporcionar una visión completa de lo realizado en el campo del conocimiento (Arksey y O’Malley, 2005).

Para llevar a cabo la revisión de literatura se siguieron los siguientes pasos sistematizados (Beltrán, 2005; Kitchenham et al., 2010): 1) clarificar el enunciado de la pregunta a responder; 2) definir los criterios de inclusión y exclusión; 3) formular el plan de búsqueda de la literatura; 4) registrar los datos y la evaluación de la calidad de los estudios seleccionados y, 5) interpretar y presentar los datos.

En la primera etapa de la planificación de la investigación, se establecieron las siguientes preguntas de investigación, a saber:

(PR1). ¿Cuáles son los países que estudian la configuración de espacios áulicos?

(PR2). ¿Qué metodologías se emplean para estudiar los espacios áulicos?

(PR3). ¿Cómo se integran las TIC en los espacios áulicos?

(PR4). ¿Qué herramientas o recursos materiales se han utilizado, en las aulas, para el aprendizaje hibrido en el contexto del COVID-19?

Para llevar a cabo la búsqueda de las fuentes se utilizaron las bases de datos de acceso abierto: SCIELO y REDALYC, así como el motor de búsqueda INVENTIO. Las palabras clave empleadas en la búsqueda fueron: espacio áulico y educación. Los tres criterios de inclusión que se tuvieron en cuenta fueron los siguientes: artículos que abordan el tema del espacio áulico de aprendizaje; publicaciones entre 2015-2021, y publicaciones realizadas en el contexto de Iberoamérica. Los criterios de exclusión que se contemplaron: ensayos, artículos teóricos, capítulos de libro y libros. En total se recopiló una base de datos de 111 artículos de los cuales se filtraron, con base en los criterios de inclusión y exclusión, quedando un total de 18 artículos seleccionados:

\section{Figura 1}

Fases de implementación de la Revisión Sistemática de Literatura

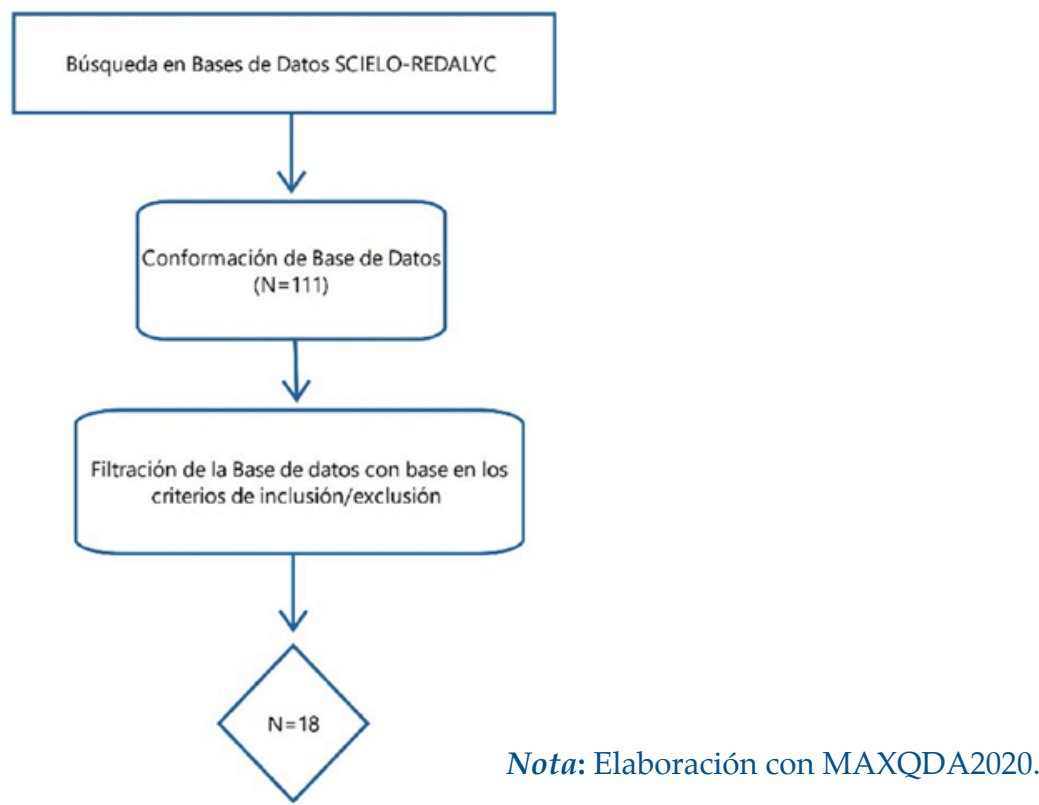




\section{RESULTADOS}

Los resultados localizados en la Revisión Sistemática de Literatura se analizaron con base en las preguntas de investigación y la estrategia de análisis de contenido cualitativo (Elo, Kääriäinen, Kanste y Pölkki, 2014). En el análisis cuantitativo se localizó la siguiente distribución de frecuencias por países, atendiendo a las preguntas de investigación:

(PR1). ¿Cuáles son los países que estudian la configuración de espacios áulicos? España (8), México (3), Colombia (3), Perú (1), Ecuador (1), Venezuela (1) y Panamá (1).

(PR2). ¿Qué metodologías se emplean para estudiar los espacios áulicos? Con relación al enfoque metodológico se encontró que nueve de los artículos son de enfoque cualitativo, seis abordaron un enfoque mixto, y tres un enfoque cuantitativo.

(PR3). ¿Cómo se integran las TIC en los espacios áulicos? Se encontró que nueve de los artículos se centraron en el espacio físico y las condiciones ambientales que favorecen el aprendizaje, cinco se apoyaron en las tecnologías de la información y las comunicaciones, y cuatro refirieron una interacción sociocultural de aprendizaje centrado en el alumno y el docente.

(PR4). ¿Qué herramientas o recursos materiales se han utilizado en las aulas para el aprendizaje hibrido, en el contexto del COVID-19? Herramientas y recursos para crear video conferencias como Zoom, Classroom, Teams. Para trabajar actividades individuales y colaborativas como el Moodle. Fomentar el aprendizaje colaborativo Office 360 y otras para presentaciones como el Genially y Prezi. De recursos materiales y equipos, tenemos: computadora, proyector, pantalla, pintarrón, equipo de sonido y pantallas interactivas.

En la Tabla 1 se presenta una síntesis de las dimensiones analizadas en los artículos de la Revisión Sistemática de Literatura.

\section{Tabla 1}

Dimensiones analizadas en la Revisión Sistemática de Literatura

\begin{tabular}{|c|c|c|c|c|c|c|}
\hline No. & Autor y año & País & Palabras clave & Objetivo & $\begin{array}{c}\text { Enfoque } \\
\text { metodológico }\end{array}$ & \multicolumn{1}{c|}{ Hallazgos } \\
\hline 1 & Casales (2021) & México & $\begin{array}{l}\text { Pedagogía, } \\
\text { aprendizaje } \\
\text { combinado, } \\
\text { planetario, } \\
\text { educación } \\
\text { inmersiva. }\end{array}$ & $\begin{array}{l}\text { Analizar el impacto } \\
\text { de los aprendizajes } \\
\text { combinados con } \\
\text { pantallas y espacio } \\
\text { físico virtual. }\end{array}$ & $\begin{array}{l}\text { Un domo de inmersión se concibe } \\
\text { como un aula educativa, donde se } \\
\text { realizan proyecciones en pantallas } \\
\text { en forma de media cúpula-y } \\
\text { donde se estimula el sentido de } \\
\text { la vista y el mundo sensorial. En } \\
\text { México se requiere la capacitación } \\
\text { docente en temas especializados } \\
\text { de esta tecnología, que busca que } \\
\text { los espacios educativos motiven } \\
\text { el aprendizaje de los estudiantes } \\
\text { (Casales, 2021). }\end{array}$ \\
\hline
\end{tabular}




\begin{tabular}{|c|c|c|c|c|c|c|}
\hline 2 & $\begin{array}{l}\text { Chávez- } \\
\text { Chiquimango } \\
\text { e Ibáñez } \\
\text { (2021) }\end{array}$ & Perú & $\begin{array}{l}\text { Comunicación } \\
\text { en salud, Perú } \\
\text { en tus manos, } \\
\text { coronavirus, } \\
\text { estudiantes } \\
\text { universitarios. }\end{array}$ & $\begin{array}{l}\text { Revisar el impacto } \\
\text { de las TIC, a través } \\
\text { de las apps que han } \\
\text { modificado la forma } \\
\text { de aprender. }\end{array}$ & Cualitativo & $\begin{array}{l}\text { El uso de las TIC y el acceso } \\
\text { a internet ha generado una } \\
\text { transformación en la forma de } \\
\text { aprender; se habilitan espacios de } \\
\text { interacción con la información, } \\
\text { y se ha incrementado el uso de } \\
\text { las apps en los teléfonos móviles. } \\
\text { Las app ayudan a la prevención y } \\
\text { comunicación de la pandemia del } \\
\text { Covid-19 (Chávez-Chiquimango } \\
\text { e Ibáñez, 2021). }\end{array}$ \\
\hline 3 & $\begin{array}{l}\text { González } \\
\text { Castro, } \\
\text { Manzano } \\
\text { Durán } \\
\text { y Torres } \\
\text { Zamudio } \\
\text { (2021) }\end{array}$ & Colombia & $\begin{array}{l}\text { Vigilancia } \\
\text { tecnológica, } \\
\text { organizaciones } \\
\text { virtuales, } \\
\text { tecnología } \\
\text { disruptiva } \\
\text { y mapa } \\
\text { tecnológico. }\end{array}$ & $\begin{array}{l}\text { Explorar las } \\
\text { tecnologías } \\
\text { disruptivas en } \\
\text { los ambientes } \\
\text { de aprendizajes } \\
\text { virtuales. }\end{array}$ & Mixto & $\begin{array}{l}\text { La educación en modalidad } \\
\text { virtual, apoyada de las nuevas } \\
\text { tecnologías disruptivas } 4.0 \\
\text { —aplicadas a las prácticas } \\
\text { académicas—, permite reconstruir } \\
\text { las actividades del aula. Uno } \\
\text { de los principales objetivos, en } \\
\text { un ambiente de aprendizaje } \\
\text { virtual, es la comunicación de los } \\
\text { participantes para la apropiación } \\
\text { del conocimiento (González } \\
\text { Castro, Manzano Durán y Torres } \\
\text { Zamudio, 2021). }\end{array}$ \\
\hline 4 & $\begin{array}{c}\text { Montoya, } \\
\text { San Juan, } \\
\text { Saavedra } \\
\text { (2020) }\end{array}$ & Colombia & $\begin{array}{l}\text { Aulas escolares, } \\
\text { desempeño } \\
\text { lumínico, clima } \\
\text { tropical, métricas } \\
\text { dinámicas, } \\
\text { simulación. }\end{array}$ & $\begin{array}{l}\text { Analizar las } \\
\text { estrategias de } \\
\text { diseño de aspectos } \\
\text { ambientales y } \\
\text { cómo incide la } \\
\text { iluminación natural } \\
\text { en el espacio. }\end{array}$ & Cuantitativo & $\begin{array}{l}\text { Presenta el análisis de las } \\
\text { estrategias de diseño para } \\
\text { alcanzar la comodidad visual } \\
\text { en las aulas y cómo inciden las } \\
\text { condiciones ambientales en } \\
\text { las actividades de aprendizaje } \\
\text { de los estudiantes. El confort } \\
\text { visual incide en la posibilidad de } \\
\text { desarrollar tareas visuales con } \\
\text { el menor riesgo posible y, por lo } \\
\text { tanto, es importante contar con } \\
\text { niveles lumínicos óptimos sobre } \\
\text { la superficie de trabajo (Montoya, } \\
\text { San Juan y Saavedra, 2020). }\end{array}$ \\
\hline 5 & $\begin{array}{c}\text { Tejedor, } \\
\text { Cervi, Tusa, } \\
\text { Parola (2020) }\end{array}$ & $\begin{array}{l}\text { España, } \\
\text { Italia y } \\
\text { Ecuador }\end{array}$ & $\begin{array}{c}\text { Tecnología } \\
\text { educativa, } \\
\text { educación } \\
\text { superior, } \\
\text { educación } \\
\text { pública, proceso } \\
\text { educativo, } \\
\text { adaptación } \\
\text { estudiantil, } \\
\text { educación } \\
\text { a distancia, } \\
\text { aprendizaje } \\
\text { virtual. }\end{array}$ & $\begin{array}{l}\text { Conocer cómo las } \\
\text { TIC han repercutido } \\
\text { en las instituciones } \\
\text { educativas y } \\
\text { mejoran el proceso } \\
\text { de aprendizaje. }\end{array}$ & Mixto & $\begin{array}{l}\text { La flexibilidad de las TIC ha } \\
\text { repercutido en las instituciones } \\
\text { educativas, el e-learning se } \\
\text { presenta como una estrategia } \\
\text { formativa para apoyar los } \\
\text { procesos educativos. Existe poca } \\
\text { capacitación docente en el uso } \\
\text { de las TIC, como herramienta } \\
\text { tecnológica, que apoya el empleo } \\
\text { socioconstructivo de la tecnología } \\
\text { educativa y el Covid-19 lo ha } \\
\text { puesto en evidencia (Tejedor, } \\
\text { Cervi, Tusa y Parola, 2020). }\end{array}$ \\
\hline
\end{tabular}




\begin{tabular}{|c|c|c|c|c|c|c|}
\hline 6 & $\begin{array}{l}\text { Fernández } \\
\text { (2020) }\end{array}$ & España & $\begin{array}{l}\text { Arquitectura, } \\
\text { Covid-19, } \\
\text { edificación, } \\
\text { urbanismo }\end{array}$ & $\begin{array}{l}\text { Analizar el } \\
\text { planteamiento de } \\
\text { la arquitectura en el } \\
\text { rediseño del espacio } \\
\text { en los edificios de } \\
\text { enseñanza y su } \\
\text { entorno para lograr } \\
\text { una interacción } \\
\text { social. }\end{array}$ & Cualitativo & $\begin{array}{l}\text { Con el confinamiento se } \\
\text { generaron modificaciones en } \\
\text { los espacios de las viviendas, } \\
\text { siendo estos más flexibles } \\
\text { para atender las actividades } \\
\text { de aprendizaje, descanso y } \\
\text { recreación. El quehacer del } \\
\text { diseño arquitectónico nos lleva } \\
\text { a replantear los espacios de uso } \\
\text { común, el aforo, las circulaciones, } \\
\text { las aulas y la vivienda para lograr } \\
\text { una interacción social (Fernández, } \\
\text { 2020). }\end{array}$ \\
\hline 7 & López (2020) & México & $\begin{array}{c}\text { El aula } \\
\text { invertida, ciclo } \\
\text { de desarrollo } \\
\text { de excelencia } \\
\text { educativa, } \\
\text { educación } \\
\text { superior, } \\
\text { fenomenológico } \\
\text { hermenéutico }\end{array}$ & $\begin{array}{l}\text { Indagar cómo se } \\
\text { incorpora el modelo } \\
\text { del aula invertida en } \\
\text { el modelo educativo } \\
\text { de educación } \\
\text { superior. }\end{array}$ & Cualitativo & $\begin{array}{l}\text { El aula invertida es un modelo } \\
\text { educativo que puede ayudar } \\
\text { a mejorar los procesos de } \\
\text { enseñanza-aprendizaje, } \\
\text { a través de la tecnología } \\
\text { educativa; el alumno necesita } \\
\text { tener conocimiento de alguna } \\
\text { tecnología educativa. Este modelo } \\
\text { no es apropiado para alumnos } \\
\text { receptores memorizadores y no } \\
\text { cuenta con un instrumento de } \\
\text { evaluación (López, 2020). }\end{array}$ \\
\hline 8 & $\begin{array}{c}\text { García Aretio } \\
\text { (2020) }\end{array}$ & España & $\begin{array}{l}\text { Educación } \\
\text { a distancia, } \\
\text { confinamiento, } \\
\text { e-learning, } \\
\text { blended-learning, } \\
\text { aprendizaje } \\
\text { híbrido, } \\
\text { combinado, } \\
\text { mixto, flexible. }\end{array}$ & $\begin{array}{l}\text { Analizar qué } \\
\text { aspectos se } \\
\text { modificarán y cómo } \\
\text { se adecuarán los } \\
\text { espacios educativos, } \\
\text { apoyados por } \\
\text { las tecnologías } \\
\text { digitales. }\end{array}$ & Cualitativo & $\begin{array}{l}\text { Al momento de surgir la } \\
\text { pandemia, muchas universidades } \\
\text { ya contaban con plataformas } \\
\text { digitales y los docentes debieron } \\
\text { agilizar su aprendizaje con } \\
\text { grandes limitaciones pedagógicas. } \\
\text { En épocas posteriores al Covid, se } \\
\text { pretende aprovechar los formatos } \\
\text { híbridos, combinados y apoyados } \\
\text { por la flexibilización del espacio } \\
\text { (García Aretio, 2020). }\end{array}$ \\
\hline 9 & $\begin{array}{c}\text { González, } \\
\text { Herrera, } \\
\text { Clerque y } \\
\text { Álvarez (2020) }\end{array}$ & Venezuela & $\begin{array}{l}\text { Aula, ambiente } \\
\text { de la clase, } \\
\text { relación } \\
\text { profesor-alumno, } \\
\text { tecnología de la } \\
\text { información. }\end{array}$ & $\begin{array}{l}\text { Conocer el } \\
\text { espacio del aula } \\
\text { y los aspectos } \\
\text { ambientales que } \\
\text { influyen para que } \\
\text { el aprendizaje sea } \\
\text { significativo. }\end{array}$ & Cuantitativo & $\begin{array}{l}\text { En los tres momentos de la clase: } \\
\text { motivación, construcción de } \\
\text { conocimiento y consolidación, } \\
\text { se revisaron varios aspectos } \\
\text { que impactan en el aula, como } \\
\text { son: la iluminación, la higiene } \\
\text { y la motivación, con los cuales } \\
\text { puede obtenerse un aprendizaje } \\
\text { significativo; pero aún falta } \\
\text { capacitar al docente en el manejo } \\
\text { de la tecnología para trabajar } \\
\text { en aulas virtuales (González, } \\
\text { Herrera, Clerque y Álvarez, } \\
\text { 2020). }\end{array}$ \\
\hline
\end{tabular}


Tabla 2

Aula de aprendizaje

\begin{tabular}{|c|c|c|c|c|c|c|}
\hline No. & Autor y año & País & Palabras clave & Objetivo & $\begin{array}{c}\text { Enfoque } \\
\text { metodológico }\end{array}$ & Hallazgos \\
\hline 10 & $\begin{array}{l}\text { Torres y González } \\
\text { (2019) }\end{array}$ & Colombia & $\begin{array}{l}\text { Espacio, } \\
\text { educación, } \\
\text { análisis } \\
\text { socioeconómico, } \\
\text { cartografía, } \\
\text { percepción. }\end{array}$ & $\begin{array}{l}\text { Analizar la relación } \\
\text { de la arquitectura } \\
\text { con la pedagogía, a } \\
\text { través del espacio } \\
\text { en las instituciones } \\
\text { educativas. }\end{array}$ & Mixto & $\begin{array}{l}\text { La relación entre la pedagogía } \\
\text { y la arquitectura encuentra } \\
\text { un trabajo interdisciplinar } \\
\text { en la forma de construir } \\
\text { espacios que cumplan con los } \\
\text { requerimientos de muebles } \\
\text { e inmuebles, y garanticen } \\
\text { las condiciones educativas. } \\
\text { La propuesta del análisis del } \\
\text { espacio educativo se basa } \\
\text { en la matriz de análisis del } \\
\text { espacio territorial, observando } \\
\text { las dimensiones espaciales } \\
\text { y territoriales (Torres y } \\
\text { González, 2019). }\end{array}$ \\
\hline 11 & $\begin{array}{c}\text { Domingo } \\
\text { Calabuig (2019) }\end{array}$ & España & $\begin{array}{l}\text { Arquitectura } \\
\text { universitaria, de } \\
\text { transición, de } \\
\text { diseño, entornos } \\
\text { de aprendizaje, } \\
\text { espacios. }\end{array}$ & $\begin{array}{l}\text { Presentar las } \\
\text { adecuaciones } \\
\text { de los espacios } \\
\text { y las novedades } \\
\text { tecnológicas que } \\
\text { se presentarán, } \\
\text { derivados de la } \\
\text { pandemia. }\end{array}$ & Cualitativo & $\begin{array}{l}\text { En la educación superior, el } \\
\text { aprendizaje semipresencial ya } \\
\text { es habitual. La transformación } \\
\text { de los entornos de } \\
\text { aprendizajes - entendidos } \\
\text { como los factores físicos, } \\
\text { virtuales y psicológicos-, } \\
\text { colocan al centro, el espacio } \\
\text { educativo y las novedades } \\
\text { tecnológicas, resaltando el } \\
\text { uso flexible del espacio y } \\
\text { considerando el aula más } \\
\text { allá de sus límites físicos } \\
\text { (Domingo Calabuig, 2019). }\end{array}$ \\
\hline 12 & $\begin{array}{c}\text { García Guardado } \\
\text { (2019) }\end{array}$ & México & $\begin{array}{l}\text { Aprendizaje } \\
\text { virtual, } \\
\text { comunicación, } \\
\text { interactividad, } \\
\text { investigación } \\
\text { documental. }\end{array}$ & $\begin{array}{l}\text { Comprender la } \\
\text { importancia de la } \\
\text { socialización en el } \\
\text { proceso de enseñanza- } \\
\text { aprendizaje, a través } \\
\text { de la interacción } \\
\text { social. }\end{array}$ & Cualitativo & $\begin{array}{l}\text { El espacio de aprendizaje } \\
\text { virtual, requiere lineamientos } \\
\text { claros que permitan la } \\
\text { interacción entre profesores } \\
\text { y alumnos; la utilización } \\
\text { de redes y aplicaciones } \\
\text { tecnológicas han favorecido } \\
\text { la comunicación. En el } \\
\text { entorno didáctico, donde } \\
\text { se utilizan las plataformas } \\
\text { para el aprendizaje a } \\
\text { distancia, se requiere de un } \\
\text { modelo de socialización con } \\
\text { implicaciones humanistas } \\
\text { (García Guardado, 2019). }\end{array}$ \\
\hline
\end{tabular}




\begin{tabular}{|c|c|c|c|c|c|c|}
\hline 13 & $\begin{array}{c}\text { Dios, Manzanares } \\
\text { y García (2018) }\end{array}$ & España & $\begin{array}{l}\text { Escenarios, } \\
\text { entornos de } \\
\text { aprendizaje, } \\
\text { future classroom } \\
\text { lab, contextos } \\
\text { de enseñanza- } \\
\text { aprendizaje, } \\
\text { competencias. }\end{array}$ & $\begin{array}{l}\text { Indagar sobre la } \\
\text { transformación del } \\
\text { aula, en un escenario } \\
\text { de aprendizaje } \\
\text { significativo. }\end{array}$ & Cualitativo & $\begin{array}{l}\text { La transformación del } \\
\text { aula en un escenario de } \\
\text { aprendizaje significativo } \\
\text { flexible, sin grandes cambios } \\
\text { estructurales, se basa en un } \\
\text { modelo de escenario junto } \\
\text { con metodologías activas, } \\
\text { como aprendizaje basado } \\
\text { en problemas-proyectos, } \\
\text { aprendizaje autorregulado, } \\
\text { aprendizaje basado en } \\
\text { indagación o flipped classroom } \\
\text { (Dios, Manzanares y García, } \\
\text { 2018). }\end{array}$ \\
\hline 14 & $\begin{array}{c}\text { Burke Mena y } \\
\text { Lasso de la Vega } \\
\text { (2017) }\end{array}$ & Panamá & $\begin{array}{l}\text { Acústica, sonido, } \\
\text { decibelio, } \\
\text { audición, } \\
\text { isosonoridad, } \\
\text { isolíneas. }\end{array}$ & $\begin{array}{l}\text { Explorar cómo } \\
\text { impacta la } \\
\text { contaminación } \\
\text { acústica en los } \\
\text { espacios educativos. }\end{array}$ & Cuantitativo & $\begin{array}{l}\text { El sonido es el contaminante } \\
\text { más común y se define como } \\
\text { el conjunto de fenómenos } \\
\text { vibratorios aéreos, percibidos } \\
\text { por el ser humano y que } \\
\text { pueden producir una reacción } \\
\text { de rechazo. El umbral de } \\
\text { audición que produce dolor, } \\
\text { se ubica alrededor de los } 120 \\
\text { dBA; el rango medio entre } \\
\text { silencioso y ruidoso, entre } \\
\text { los } 55 \text { dBA y } 70 \text { dBA. Se } \\
\text { recomienda el monitoreo de } \\
\text { las fuentes emisoras de ruido } \\
\text { (Burke Mena y Lasso de la } \\
\text { Vega, 2017). }\end{array}$ \\
\hline 15 & $\begin{array}{c}\text { Pisá Bó y } \\
\text { Novejarque } \\
\text { Civera (2017) }\end{array}$ & España & $\begin{array}{l}\text { TIC, tabletas, } \\
\text { metodologías } \\
\text { activas de } \\
\text { aprendizaje, } \\
\text { educación } \\
\text { primaria, } \\
\text { educación } \\
\text { secundaria. }\end{array}$ & $\begin{array}{l}\text { Identificar aspectos } \\
\text { que permitan mostrar } \\
\text { las ventajas de las } \\
\text { TIC en el aula, y la } \\
\text { interacción entre el } \\
\text { alumno y el docente. }\end{array}$ & Cualitativo & $\begin{array}{l}\text { Las ventajas de las TIC en el } \\
\text { aula, radican en que ofrecen } \\
\text { posibilidades de mejora } \\
\text { del aprendizaje, ya que } \\
\text { aportan diversos recursos y } \\
\text { actividades que logran hacer } \\
\text { que este sea más atractivo } \\
\text { y motivador. Una de sus } \\
\text { principales ventajas es el } \\
\text { acceso a los contenidos en } \\
\text { línea (Pisá Bó y Novejarque } \\
\text { Civera, 2017). }\end{array}$ \\
\hline
\end{tabular}




\begin{tabular}{|c|c|c|c|c|c|c|}
\hline 16 & $\begin{array}{c}\text { García-Cano, } \\
\text { Hinojosa Pareja } \\
\text { y Gutiérrez } \\
\text { Santiuste (2017) }\end{array}$ & España & $\begin{array}{l}\text { Innovaciones } \\
\text { educativas, } \\
\text { educación } \\
\text { superior, } \\
\text { formación de } \\
\text { profesores, } \\
\text { organizaciones } \\
\text { sociales, } \\
\text { participación } \\
\text { social. }\end{array}$ & $\begin{array}{l}\text { Explorar la } \\
\text { relación entre las } \\
\text { organizaciones } \\
\text { sociales, en el } \\
\text { desarrollo de la } \\
\text { actividad docente y } \\
\text { la construcción de } \\
\text { aprendizajes. }\end{array}$ & Mixto & $\begin{array}{l}\text { A lo largo de la innovación } \\
\text { docente, se ha encontrado } \\
\text { una evolución en la } \\
\text { importante reducción de } \\
\text { la falta de motivación, } \\
\text { el desconocimiento o } \\
\text { la oportunidad para no } \\
\text { participar en organizaciones } \\
\text { sociales; el desarrollo de } \\
\text { una formación docente, } \\
\text { comporta nuevas propuestas } \\
\text { pedagógicas flexibles y } \\
\text { permeables (García-Cano, } \\
\text { Hinojosa Pareja y Gutiérrez } \\
\text { Santiuste, 2017). }\end{array}$ \\
\hline 17 & $\begin{array}{c}\text { Rodríguez, } \\
\text { Gallego y } \\
\text { Rodríguez, V. } \\
\text { (2016) }\end{array}$ & España & $\begin{array}{c}\text { Espacios } \\
\text { arquitectónicos } \\
\text { en la formación } \\
\text { profesional, } \\
\text { potencial del } \\
\text { espacio en la } \\
\text { generación } \\
\text { de relaciones, } \\
\text { permeabilidad } \\
\text { en las aulas, gran } \\
\text { taller. }\end{array}$ & $\begin{array}{l}\text { Indagar sobre las } \\
\text { características } \\
\text { de diseño de los } \\
\text { espacios educativos, } \\
\text { y la generación e } \\
\text { interacción en los } \\
\text { centros de formación. }\end{array}$ & Cualitativo & $\begin{array}{l}\text { Sobre la importancia de las } \\
\text { características arquitectónicas } \\
\text { del centro educativo, } \\
\text { prevalecen las condiciones } \\
\text { físico-ambientales, } \\
\text { como la iluminación y la } \\
\text { ventilación; además, el que } \\
\text { los espacios cuenten con } \\
\text { mayor flexibilidad para la } \\
\text { adecuación de proyectos } \\
\text { educativos emergentes y el } \\
\text { diseño de espacios vinculados } \\
\text { que fomenten la interacción } \\
\text { física entre docentes y } \\
\text { alumnos (Rodríguez, Gallego } \\
\text { y Rodríguez, V., 2016). }\end{array}$ \\
\hline 18 & $\begin{array}{c}\text { Aguilar Ramos y } \\
\text { Linde Valenzuela } \\
\text { (2015) }\end{array}$ & España & $\begin{array}{l}\text { Innovación, } \\
\text { competencias, } \\
\text { aprendizaje, } \\
\text { formación } \\
\text { pedagógica. }\end{array}$ & $\begin{array}{l}\text { Indagar sobre el } \\
\text { impacto de las TIC } \\
\text { en la innovación en el } \\
\text { aula y los espacios de } \\
\text { aprendizaje. }\end{array}$ & Mixto & $\begin{array}{l}\text { La experiencia innovadora en } \\
\text { la práctica docente, se centra } \\
\text { en las TIC y el uso de recursos } \\
\text { tecnológicos digitales, creando } \\
\text { escenarios de aprendizaje. } \\
\text { La intervención innovadora } \\
\text { se basa en la utilización de } \\
\text { un recurso, un diseño o una } \\
\text { planificación de una situación } \\
\text { novedosa en el aula; se ha } \\
\text { buscado que el proceso de } \\
\text { enseñanza-aprendizaje sea } \\
\text { dinámico para poder llevarlo } \\
\text { a la realidad (Aguilar Ramos } \\
\text { y Linde Valenzuela, 2015). }\end{array}$ \\
\hline
\end{tabular}




\section{Disposición y adecuaciones tecnológicas de las aulas en el contexto COVID-19}

En este eje temático se agruparon aquellas investigaciones que documentaron adecuaciones en los espacios áulicos. La investigación de Casales-Navarrete (2021) resaltó que la capacitación docente, en temas especializados de tecnología, busca que los espacios educativos motiven el aprendizaje de los estudiantes; por la pandemia de COVID-19 se ha incrementado el uso de pantallas. De igual manera, Chávez-Chiquimango e Ibáñez (2021) mencionan que el uso de las TIC y el acceso a internet, generaron cambios en la forma de aprender y se ha incrementado el empleo de las apps en teléfonos móviles inteligentes, y que ayudan a la prevención y transmisión de la pandemia.

La flexibilidad de las TIC ha conducido a un mayor uso de internet. Además, el e-learning, con su componente de interactividad, se presenta como una estrategia formativa para los procesos educativos (Tejedor, Cervi, Tusa y Parola, 2020). Uno de los principales objetivos en un ambiente de aprendizaje virtual, es la comunicación de los participantes para la apropiación del conocimiento, ya que permite reconstruir las actividades en el aula (González, Manzano y Torres, 2021).

Menciona Fernández (2020) que antes de la pandemia ya se contaba con actividades docentes formativas en línea y, con el confinamiento, el diseño arquitectónico nos llevó a replantear los espacios de uso común y las aulas para lograr mayor interacción social; se contaba además con plataformas digitales y los docentes tuvieron que agilizar su aprendizaje con limitaciones pedagógicas. En épocas posteriores al COVID-19, se pretende aprovechar los modelos híbridos y combinados.

El «aula invertida» es un modelo educativo que puede ayudar a mejorar los procesos de enseñanza-aprendizaje a través de la tecnología educativa (López, 2020); para Domingo Calabuig (2019), el aprendizaje semipresencial en la educación superior es ya más habitual, se resalta el uso flexible del espacio, los materiales y los espacios abiertos, por lo que debe considerarse el aula más allá de sus límites físicos.

El espacio de aprendizaje virtual requiere lineamientos claros que permitan la interacción entre profesores y alumnos, y establecer un modelo de socialización a través de la tecnología con implicaciones humanistas, señala García (2019). Para Pisá y Novejarque (2017), las ventajas de las TIC en el aula ofrecen posibilidades de mejora del aprendizaje, ya que aportan diversos recursos y actividades que logran hacer que este sea más atractivo y motivador. Por otro lado, Aguilar Ramos y Linde Valenzuela (2015), mencionan que la experiencia innovadora en la práctica docente, se centra en las TIC en el ámbito de recursos tecnológicos digitales, buscando que el proceso de enseñanza-aprendizaje sea dinámico. El desarrollo de una formación docente -desde la premisa de motivaciones intrínsecas y vivenciales-, comporta nuevas propuestas pedagógicas flexibles y permeables (García-Cano, Hinojosa, Gutiérrez, 2017).

\section{Interacción entre arquitectura y pedagogía en la construcción de espacios áulicos}

Se presentó el análisis socioespacial de una institución educativa y se encontró que la arquitectura y la pedagogía se encuentran en un trabajo interdisciplinar en la forma de construir espacios (Torres y González, 2019). Las características arquitectónicas del centro 
educativo que prevalecen son las condiciones físico-ambientales, como la iluminación y la ventilación, aunado a la flexibilidad de los espacios que fomentan la interacción física entre los docentes y los alumnos (Rodríguez, Gallego y Rodríguez, 2016).

Para González, Herrera, Clerque y Álvarez (2020), en los tres momentos de la clase, los aspectos que impactan en el aula son: iluminación, higiene y motivación, pero aún falta capacitar al docente en el manejo de tecnologías para aulas virtuales. Otra condición ambiental que incide en las actividades de aprendizaje es el confort visual, por lo que debe contarse con niveles óptimos lumínicos (Montoya, San Juan y Saavedra, 2020). Señala Burke (2017) que el sonido es el contaminante más común percibido por el ser humano y puede producir una reacción de rechazo, por lo que se recomienda dar seguimiento al estudio para mejorar el monitoreo de las fuentes emisoras de ruido. Por último, se realizó una propuesta de transformación del aula en un escenario de aprendizaje significativo que, sin necesidad de grandes cambios estructurales y con metodologías activas, tenga un impacto positivo en la motivación del alumno (Dios, Manzanares y García, 2018).

\section{DISCUSIÓN}

Con base en los hallazgos reportados y el objetivo de investigación, que giró en torno a la indagación de artículos empíricos sobre el espacio áulico, pueden discutirse los siguientes tópicos:

1) Los retos que enfrentan diversos países en torno a la modificación de los espacios áulicos para el cumplimiento de las medidas sanitarias en COVID-19.

2) El uso de tecnologías educativas para la adecuación de los espacios áulicos.

3) La capacitación docente en el uso de los espacios bajo el modelo híbrido.

4) La configuración arquitectónica del aula. Por ejemplo, la iluminación dentro como una condición ambiental, incide en las actividades de aprendizaje, directamente en el confort visual, por lo que debe contarse con los niveles lumínicos óptimos; de igual manera, la calidad de la ventilación influye directamente en este proceso.

El rediseño de espacios arquitectónicos, en el contexto COVID-19 para los centros educativos, se ha analizado extensivamente, tanto en las aulas como en otros espacios, por ejemplo, las bibliotecas (Vassilakaki y Moniarou-Papaconstantinou, 2021). En dichas investigaciones se recomienda evaluar los espacios físicos y comenzar proyectos de rediseño paulatinos en los que se valoren elementos como la acústica, dentro del espacio áulico, y la ventilación de los espacios.

En las investigaciones, localizadas a través de la revisión sistemática de literatura, se observa un uso mayoritario de enfoques cualitativos de investigación, lo cual es un aporte importante en la comprensión de la construcción de los aprendizajes dentro de los espacios áulicos. Como lo mencionan diferentes teóricos del socio-constructivismo, el aprendizaje es co-construido y para ello se requiere no solo de un espacio físico, sino de estrategias que promuevan la reflexión, la creatividad y el despliegue de competencias (Masciotra y Medzo, 2009; Perrenoud, 2008).

Finalmente, se discute la inclusión de estrategias de enseñanza innovadoras que puedan implementarse dentro de los espacios áulicos rediseñados (Ramírez-Ramírez y Ramírez-Montoya, 2018) y que favorezcan los aprendizajes significativos de los estudiantes, manteniendo los elementos de colaboración, flexibilidad, reflexión y pensamiento crítico que se requieren en el contexto actual (Meek, Teichler y Kearney, 2009). 


\section{CONCLUSIÓN}

Como recomendación para la línea de investigación, sobre adecuaciones a los espacios áulicos, se sugiere el desarrollo de lineamientos en los centros educativos que permitan orientar en el rediseño, la construcción, la implementación y el uso de los espacios áulicos para la adaptación a las nuevas necesidades en tiempos de post-pandemia.

Dentro de la estrategia a seguir, en período post-pandemia, está la de equipar las aulas con más herramientas digitales -como software, plataformas didácticas- elementos acústicos y mejorar la ventilación con medios naturales o mecánicos, buscando que todo ello ayude a la motivación del estudiante y fortalezca los aprendizajes significativos. Definitivamente la capación docente es fundamental, junto con las estrategias pedagógicas que deben ir muy de la mano: la pandemia COVID-19 puso en evidencia que el sistema educativo requiere de una estrategia educativa virtual.

Si bien se atendió la emergencia utilizando plataformas virtuales de aprendizaje, se patentizó asimismo la capacitación docente en el manejo de herramientas tecnológicas, es por ello que se pretende implementar el uso de las aulas híbridas que ofrecen clases presenciales y en línea, y fortalecer las herramientas tecnológicas.

\section{REFERENCIAS BIBLIOGRÁFICAS}

Aguilar Ramos, M. y Linde Valenzuela, T. (2015). Innovación en el aula: creación de espacios de aprendizaje. Hekademos. Revista Educativa Digital, 17, 93-103.

Arksey, H. \& O’Malley, L. (2005). Scoping Studies: Towards a Methodological Framework. International Journal of Social Research Methodology, 8(1), 19-32.

Bó, M. P. y Civera, J. N. (2017). Las TICs en el aula y su efecto final en el resultado de aprendizaje. En La innovación docente como misión del profesorado. Actas del IV Congreso Internacional sobre Aprendizaje, Innovación y Competitividad/CINAIC (pp. 689-694). Universidad de Zaragoza.

Beltrán G. y Óscar A. (2005). Revisiones sistemáticas de la Literatura. Revista Colombiana de Gastroenterología, 20(1), 60-69 http://www.redalyc.org/articulo. oa?id=337729264009.

Burke Mena, G. (2017). Contaminación Acústica en el campus de la USMA. Investigación y Pensamiento Crítico, 5(3), 19-33. https:// doi.org/10.37387/ipc.v5i3.73

Cabrero Almenara, J. y Martínez-Gimeno, A. (2019). Las Tecnologías de la Información y Comunicación y la Formación Inicial de los Docentes. Modelos y Competencias Digitales. Profesorado, Revista de Currículum y Formación del Profesorado, 23(3), 247-268. htttps: / / doi.org/10.30827/ profesorado.v23i3.9421

Casales Navarrete, A. (2021). Experiencias educativas con aprendizajes combinados y un domo de inmersión, un estudio de caso. Tecnología \& Diseño, 15, 27-47. https:// revistatd.azc. uam.mx/index.php/rtd/article/view/99/204 
CEPAL-UNESCO.(2020).LaeducaciónentiemposdelapandemiaCOVID-19.ComisiónEconómica para América Latina y El Caribe, 11-13. https:/ / unesdoc.unesco.org/ark:/ 48223/ pf0000374075? posInSet=1\&queryId=9ccf4a39-7c50-43e4-856b-a09632daa7a2

Chávez-Chiquimango, M. A. e Ibáñez, D. B. (2021). El uso de apps durante la pandemia de COVID-19: Un estudio de caso a partir de estudiantes universitarios de Lima (Perú). RISTI - Revista Iberica de Sistemas e Tecnologias de Informação, 2021(E40), 51-63.

Dios, M. A. Q., Manzanares, M. C. S. y García, E. M. (2018). Transformar el aula en un escenario de aprendizaje significativo. Hekademos. Revista Educativa Digital, 24, 7-18.

Domingo Calabuig, D. (2019). Espacios intermedios, espacios de relación, espacios para el aprendizaje: lecturas de transición en la arquitectura universitaria. Eídos, 14(diciembre), 89-98. https:// doi.org/10.29019/eidos.v14i1.610

Elo, S., Kääriäinen, M., Kanste, O. \& Pölkki, T. (2014). Qualitative Content Analysis: A Focus on Trustworthiness. Sage Open, January-Ma, 1-10. https://doi. org/10.1177/2158244014522633

Fernández, M. (2020). La influencia de la arquitectura y el diseño del espacio en la enseñanza post COVID-19. Covid-19. Educación Inclusiva y Personas con Discapacidad: Fortalezas y Debilidades de la Teleeducación, 1, 45-53. http://hdl.handle.net/11181/6156

García-Cano, M., Hinojosa Pareja, E. y Gutiérrez Santiuste, E. (2017). Construcción de Aprendizajes Académicos y Ciudadanos más allá del Aula. Revista Mexicana de Investigación Educativa, 22(74), 889-921.

García Aretio, L. (2020). COVID-19 y educación a distancia digital: preconfinamiento, confinamiento y posconfinamiento. RIED. Revista Iberoamericana de Educación a Distancia, 24(1), 09. https:// doi.org/10.5944/ried.24.1.28080

García Guardado, E. de J. (2019). La interactividad en espacios de aprendizaje virtuales: roles de profesores y estudiantes. Daena: International Journal of Good Conscience, 14(1), 5-29.

González Castro, Y., Manzano Duran, O. y Torres Zamudio, M. (2021). Tecnologías disruptivas en educación virtual. Revista Boletín Redipe, 10(7), 185-200. https://doi. org/10.36260/rbr.v10i7.1357

González, R. C. V., Herrera, D. G. G., Clerque, S. E. M. y Álvarez, J. C. E. (2020). Ejecución de una clase dentro del aula y en la virtualidad. Revista Arbitrada Interdiciplinaria Koinonía, 5(1), 392-410. http:/ / dx.doi.org/10.35381/r.k.v5i1.789

Ibero (2021). Desigualdad educativa, que ahondó la pandemia, requiere políticas públicas. https:/ / ibero. $\mathrm{mx} /$ prensa/desigualdad-educativa-que-ahondo-la-pandemia-requiere-politícas-públicas

INEGI (2021). Encuesta par la Medición del Impacto COVID-19 en la Educación (ECOVID-ED), $1-27$.

Kitchenham, B., Pretorius, R., Budgen, D., Brereton, O. P., Turner, M., Niazi, M. \& Linkman, S. (2010). Systematic Literature Reviews in Software Engineering - A Tertiary Study. Information and Software Technology, 52(8), 792-805. https:// doi.org/10.1016/j. infsof.2010.03.006 
López, E. P. M. (2020). Limitaciones en el uso del aula invertida en la educación superior. Revista Transdigital, 1(1), 1-28.

Lloyd, M. (2020). Desigualdades educativas y la brecha digital en tiempos de COVID-19. La Educación y pandemia: una visión académica (pp.115-121). México: Instituto de Investigaciones sobre la Universidad y la Educación. https://n9.cl/o4zcg

Masciotra, D. \& Medzo, F. (2009). Déveloper un agir compétent. Vers un curriculum pour la vie. De Boeck Supérieur. https:/ / doi.org/10.3917/ dbu.masci.2009.01

Meek, V. L., Teichler, U. \& Kearney, M. (2009). Higher Education, Research and Innovation: Changing Dynamics. Report on the UNESCO Forum on Higher Education, Research and Knowledge 2001-2009. UNESCO/International Centre for Higher Education Research Kassel.

Méndez Escobar, A. (2021). Educación en tiempos de pandemia (COVID-19). Revista de la Universidad de la Salle, 2020(85), 51-59.

Mohamed Al-Lal, F. (2021). Aula inteligente: Definición y evolución. Revista Didasc@lia: Didáctica y Educación, 12(2), 1-24.

Montoya, O., San Juan, G. A. y Saavedra, L. (2019). Evaluación de propuestas de diseño para la optimización del desempeño lumínico en aulas del trópico, basadas en métricas dinámicas. Avances en Energías Renovables y Medio Ambiente, 23.

Perrenoud, P. (2008). Diez nuevas competencias para enseñar. Tiempo de Educar, 9(17), 159.

Pisá-Bó, M. P. y Novejarque-Civera, J. N. (2017). Las TICs en el aula y su efecto final en el resultado de aprendizaje. La innovación docente como misión del profesorado. Actas del IV Congreso Internacional sobre Aprendizaje, Innovación y Competitividad, CINAIC 2017 (pp. 689-694). Universidad de Zaragoza.

Prince Torres, A. C. (2021). Aulas híbridas: Escenarios para transformación educativa dentro de la nueva normalidad. Podium, 39, 103-120. https:/ / doi.org/10.31095/podium.2021.39.7

Ramírez-Ramírez, L. N., y Ramírez-Montoya, M. S. (2018). El papel de las estrategias innovadoras en educación superior: retos en las sociedades del conocimiento. Revista Pedagogía, 39(104), 147-170. https:// repositorio.itesm.mx/bitstream/handle/11285/630729/Ramírez\%26RamírezUpedagocia.pdf?sequence=1\&isAllowed=y

Rodríguez, L., Gallego, J. L. y V. Rodríguez, A. (2016). Reflexiones docentes acerca del diseño arquitectónico de los centros de formación profesional en Granada. Propósitos y Representaciones, 4(1) 115-168. https: / / doi.org/10.20511/pyr2016.v4n1.88

Schmelkes, S. (2020). La educación superior ante la pandemia de la COVID-19: el caso de México. Universidades, 71(86), 73-87.

Steelcase (2021). Designing Post-COVID Learning Spaces. https://www.steelcase.com/ research/articles/topics/education/designing-post-covid-learning-spaces / 
Torres, D. R. V. y González, S. L. A. (2019). Análisis socio espacial de las instituciones educativas: estudio de caso del territorio escolar. Educación y Territorio, 9(16), 13-46. https:// doi.org/10.38017/22563989.702

Tejedor, S., Cervi, L., Tusa, F. y Parola, A. (2020). Educación en tiempos de pandemia: Reflexiones de alumnos y profesores sobre la enseñanza virtual universitaria en España, Italia y Ecuador. Revista Latina de Comunicación Social, 78, 1-21. https: / / www. doi.org/10.4185/RLCS-2020-1466

UNICEF (2021). Guidance for COVID-19. Prevention and Control in Schools. http:/ / www. unicef. org/lac/media/12481/ file

Vassilakaki, E. \& Moniarou-Papaconstantinou, V. (2021). Library Space and COVID-19: Re-thinking of Place and Re-designing of Digital Space. In Libraries, Digital Information, and COVID (pp. 207-213). Chandos Publishing.

Xiao, Y. \& Watson, M. (2019). Guidance on Conducting a Systematic Literature Review. Journal of Planning Education and Research, 39(1), 93-112. Retrieved from https:// journals.sagepub.com/doi/full/10.1177/0739456X17723971 